$$
\text { Conf-950226--54 }
$$

UCRL-JC-119611

PREPRINT

\title{
Amplification of Femtosecond Pulses to Above 1 J with Large Aperture Cr:LiSrAIF Amplifiers
}

\author{
T. Ditmire \\ M. D. Perry
}

This paper was prepared for submittal to the SPIE's 1995 International Symposium on Lasers and Applications San Jose, CA

February 4-10, 1995

January 1995

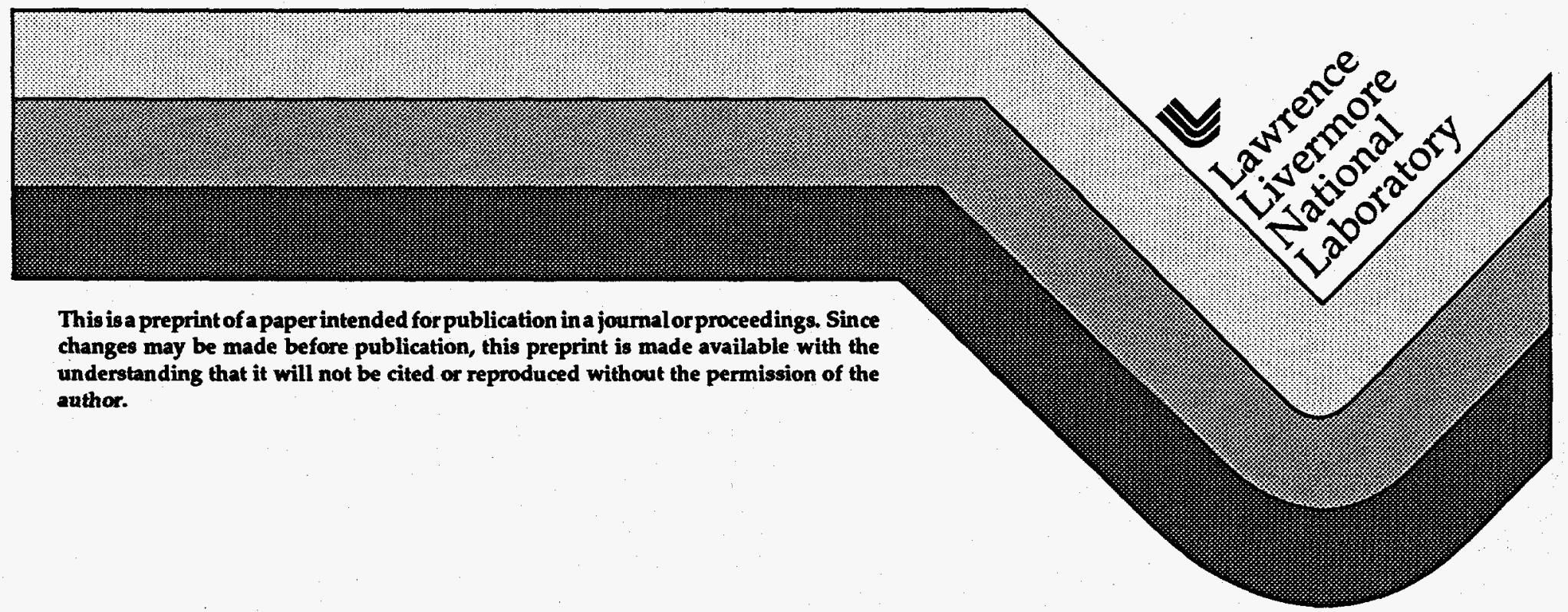<smiles>[AlH2]</smiles> 


\section{DISCLAIMER}

This document was prepared as an account of work sponsored by an agency of the United States Government. Neither the United States Government nor the University of California nor any of their employees, makes any warranty, express or implied, or assumes any legal liability or responsibility for the accuracy, completeness, or usefulness of any information, apparatus, product, or process disclosed, or represents that its use would not infringe privately owned rights. Reference herein to any specific commercial product, process, or service by trade name, trademark, manufacturer, or otherwise, does not necessarily constitute or imply its endorsement, recommendation, or favoring by the United States Government or the University of California. The views and opinions of authors expressed herein do not necessarily state or reflect those of the United States Government or the University of California, and shall not be used for advertising or product endorsement purposes. 


\section{DISCLAIMER}

Portions of this document may be illegible in electronic image products. Images are produced from the best available original document. 


\title{
Amplification of Femtosecond Pulses to Above $1 \mathrm{~J}$
}

\author{
With Large Aperture Cr:LiSrAlF 6 Amplifiers
}

\author{
T. Ditmire, and M. D. Perry
}

Laser Program, Lawrence Livermore National Laboratory

P.O. Box 808, L-443

Livermore, CA 94550

\begin{abstract}
We have developed a chirped pulse amplification system capable of producing femtosecond pulses with energy above one joule. This is accomplished by using a large aperture, flashlamp pumped $\mathrm{Cr}$ :LiSrAlF6 (Cr:LiSAF) amplifier. Optimum design of the $19 \mathrm{~mm}$ diameter amplifier results in a single pass gain of 5 with good beam quality. This amplifier produces $1.05 \mathrm{~J}$ pulses after compression with a width of $<125 \mathrm{fs}$ at a repetition rate of $0.05 \mathrm{~Hz}$.
\end{abstract}

\section{Introduction}

The generation of high energy femtosecond pulses by chirped pulse amplification requires a broad bandwidth amplifying medium. The development of the solid state laser material, $\mathrm{Cr}_{\text {:LiSrAIF }}$ (LiSAF) ${ }^{1}$, offers an important alternative for short pulse laser applications over the material most commonly used in such lasers, Ti:sapphire. Like Ti:sapphire, LiSAF exhibits a broad bandwidth (tunable from 780 to $980 \mathrm{~nm}$ ). Unlike Ti:sapphire, however, it has a long upper state lifetime (67 $\mu s)$ allowing it to be directly flashlamp pumped. LiSAF also exhibits the attractive properties of low thermal lensing and a low nonlinear refractive index ( $5 \times 10^{-14}$ esu). The long upper state lifetime makes possible the development of compact, high energy ( $>1 \mathrm{~J}$ ) femtosecond laser systems by utilizing large aperture flashlamp pumped amplifiers. To date. systems employing Ti:sapphire have been limited to pulses of energy $<300 \mathrm{~mJ}$ and peak power of a few terawatts $(<5)$ because of the necessity of pumping by a secondary laser. $2,3,4$

Small scale flashlamp-pumped Cr:LiSAF lasers were first demonstrated by Stalder, 5 followed immediately by the development of femtosecond systems utilizing flashlamp-pumped Cr.LiSAF amplifiers. ${ }^{6,7}$ Hybrid systems employing Ti:sapphire for the production of low energy pulses and Cr:LiSAF for amplification to the $100 \mathrm{~mJ}$ level have been demonstrated as well. ${ }^{8}$ Pulse energy as high as $750 \mathrm{~mJ}$ has been demonstrated in a femtosecond Cr:LiSAF system employing a $25 \mathrm{~mm}$ diameter flashlamp-pumped amplifier. ${ }^{9}$ This system employed two $25 \times 115 \mathrm{~mm} \mathrm{Cr}: \mathrm{LiSAF}$ rods placed end to end pumped with $5 \mathrm{~kJ}$ of electrical energy to produce a single pass gain of 2.4 with a maximum repetition rate of 1 pulse every 10 minutes. ${ }^{10}$ In this letter, we report the development of a flashlamp-pumped $\mathrm{Cr}: \mathrm{LiSAF}$ system producing femtosecond, $1 \mathrm{~J}$ pulses at a repetition rate of one shot every 20 seconds. The system utilizes a single $19 \times 110 \mathrm{~mm}$ amplifier exhibiting a single pass gain of 5 to produce $1.7 \mathrm{~J}$ pulses before compression. Recompression after amplification yields $125 \mathrm{fs}$, transform limited pulses with a pulse energy of $1.05 \mathrm{~J}$ and geod beam quality.

\section{Laser Front End}

The optical layout of the LiSAF system is shown in figure 1. A Kerr lens mode-locked Ti:sapphire oscillator produces $110 \mathrm{fs}, 825 \mathrm{~nm}$ pulses which are passed four times through an $1800 \mathrm{line} / \mathrm{mm}$ grating pair pulse stretcher yielding positively 


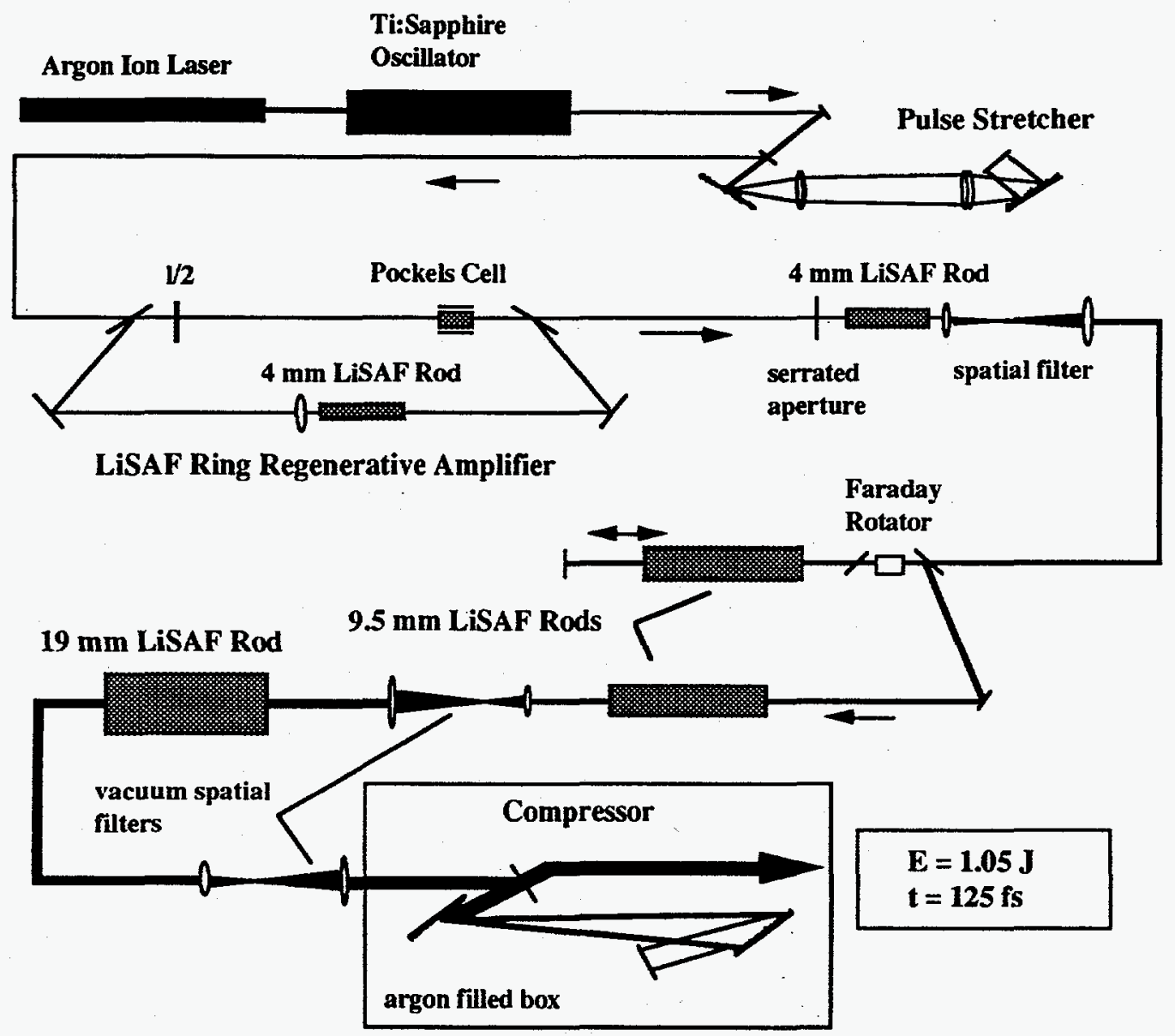

Figure 1: Schematic layout of the $1 \mathrm{~J}, 125 \mathrm{fs}$ Cr:LiSAF laser system.

chirped 450 ps pulses. A large stretching ratio $\left(t_{p} \geq 0.5 \mathrm{~ns}\right)$ is desirable to limit the nonlinear phase accumulated through the amplifier chain which can severely distort the recompressed pulse shape. ${ }^{11}$ The $\sim 1 \mathrm{~nJ}$ pulses then enter a TEM00 stable ring regenerative amplifier. ${ }^{12}$ The regenerative amplifier utilizes a single $4 \mathrm{~mm}$ flashlamp pumped LiSAF amplifier which has a single pass gain of 3. The pulse transits the regenerative amplifier 35 times before it is switched out, yielding a net gain of > $10^{7}$ and a pulse energy of $>12 \mathrm{~mJ}$. The pulse is then passed through a serrated aperture which truncates the wings of the Gaussian spatial profile. The image of this aperture is then relayed with magnification through the remainder of the amplifier system resulting in a near super-Gaussian beam at the output. Flattop profiles permits a greater fill in the amplifiers and results in greater energy extraction. A second $4 \mathrm{~mm}$ LiSAF amplifier amplifies the pulse after the serrated aperture, and an air spatial filter expands the beam to $6.5 \mathrm{~mm}$.

The pulse is then amplified in two $9.5 \mathrm{~mm}$ LiSAF amplifiers. We have optimized the performance of our large LiSAF flashlamp pumped heads to increase the maximum single pass gain. ${ }^{13}$ These amplifiers each utilize a flashlamp pulse forming network that delivers a $140 \mu \mathrm{s}$ flashlamp pulse. By shortening the flashlamp pulse width to more closely match the upper state lifetime of the LiSAF we have achieved electrical to stored energy efficiencies of $>1.4 \%$ in these amplifiers. ${ }^{13}$ Both amplifiers exhibit very good gain uniformity across the aperture of the rod. The first $9.5 \mathrm{~mm}$ rod is double passed and the second is single passed, yielding a net gain of 110 . These amplifier rods are each pumped in a cavity with four flashlamps by $600 \mathrm{~J}$ of electrical energy. The energy at this stage in the system is $400 \mathrm{~mJ}$. A vacuum spatial filter then expands the beam to $14 \mathrm{~mm}$ for amplification in the $19 \times 110 \mathrm{~mm}$ LiSAF amplifier. 


\section{Performance and Modeling of the $19 \mathrm{~mm}$ LiSAF Amplifier}

To bring the pulse energy to above one joule in a femtosecond system a relatively large aperture amplifier $(\sim 2 \mathrm{~cm}$ diameter) is required. By using a relatively short rod (length $\leq 11 \mathrm{~cm}$ ) we can obtain LiSAF of high optical quality with clear apertures of $>20 \mathrm{~mm}$. The amplifier head for our $19 \mathrm{~mm}$ rod uses 8 lamps in a cylindrical pump cavity and delivers up to $3 \mathrm{~kJ}$ of electrical pump energy in a $160 \mu \mathrm{s}$ flashlamp pulse. The gain of this amplifier for a full aperture beam (14 $\mathrm{mm})$ as a function of input electrical energy is shown in figure 2. At low pump energies, the pump efficiency is high (1.6\%), but at thehigher * pump energies this efficiency begins to drop. The result is that the small signal gain grows roughly linearly with increasing pump energy.

We attribute this drop in the pump efficiency to the shift of the Xe flashlamp light spectrum toward the ultraviolet with increasing electrical pump energy. This shifts the flashlamp light away from the LiSAF absorption bands and lowers the excitation efficiency. To model this effect, we use the fact that the spectral characteristics of small bore Xe flashlamps can be well approximated by that of a plasma column emitting radiation with a single effective blackbody temperature ${ }^{14,15^{\circ}}$ (in ${ }^{\circ} \mathrm{K}$ ) given by: 16,17

$T(J)=9300 J^{0.18} D^{0.092} P^{-0.017}$

where $\mathrm{D}$ is the Xe plasma column diameter in centimeters and $\mathrm{P}$ is the $\mathrm{Xe}$ fill pressure in torr ( $=800$ torr for our lamps). The actual emitted flashlamp spectrum is given by the relation:

$$
I(\lambda)=I_{B B}(\lambda, T) F(\alpha(\lambda) d)
$$

where $I_{B B}(\lambda, T)$ is the black-body spectrum at a temperature $T, \alpha(\lambda)$ is the wavelength dependent absorption of the Xe plasma and $d$ is the Xe plasma diameter (taken to be $5 \mathrm{~mm}$ for our lamps). $F(\alpha(\lambda) d)$ is the angle averaged emissivity function of the Xe flashlamps and has been calculated in reference 17. For the parameters that are relevant for the $19 \mathrm{~mm}$ LiSAF head, the emissivity is generally quite flat over the wavelength range of interest for LiSAF pumping. The time dependent current density, $\mathrm{J}$ (in $\mathrm{kA} / \mathrm{cm}^{2}$ ), is calculated from the time dependent behavior of the LRC circuit which produces the flashlamp pulse. ${ }^{18}$ The effect of the UV shifting of the flashlamp spectrum is illustrated in figure 3 . Here the calculated flashlamp emission spectra for plasma temperatures of $13000 \mathrm{~K}$ and $19000 \mathrm{~K}$ are compared to the absorption spectrum of the LiSAF. These temperatures correspond to the spectra at the peak of the flashlamp discharge output for 60 and $350 \mathrm{~J}$ discharged per lamp in a $160 \mu$ s pulse respectively. As this figure illustrates, the bulk of the flashlamp emission is pushed away from the primary LiSAF absorption band at $425 \mathrm{~nm}$ toward the blue at the higher pump energy.

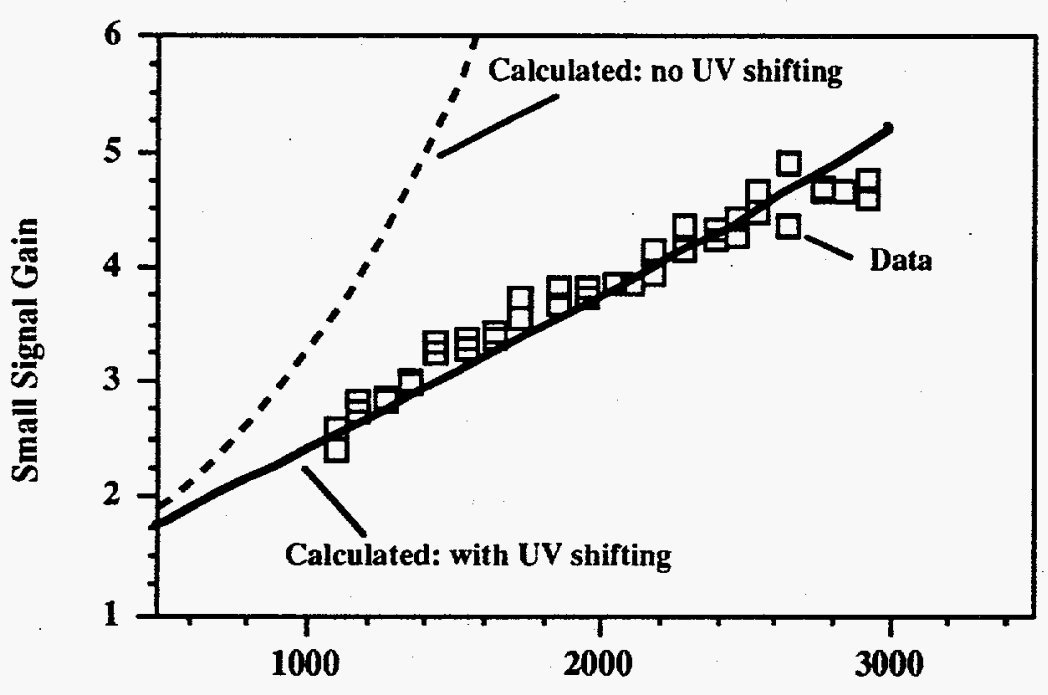

Electrical Pump Energy (J)
Figure 2: Measured small signal gain in the $19 \mathrm{~mm}$ LiSAF amplifier as a function of electrical pump energy discharged through the flashlamps (squares). The dashed line is the calculated small signal gain assuming a constant $1.8 \%$ pump efficiency and the solid line is the calculated gain when the UV shift of the Xe spectrum is taken into account. 


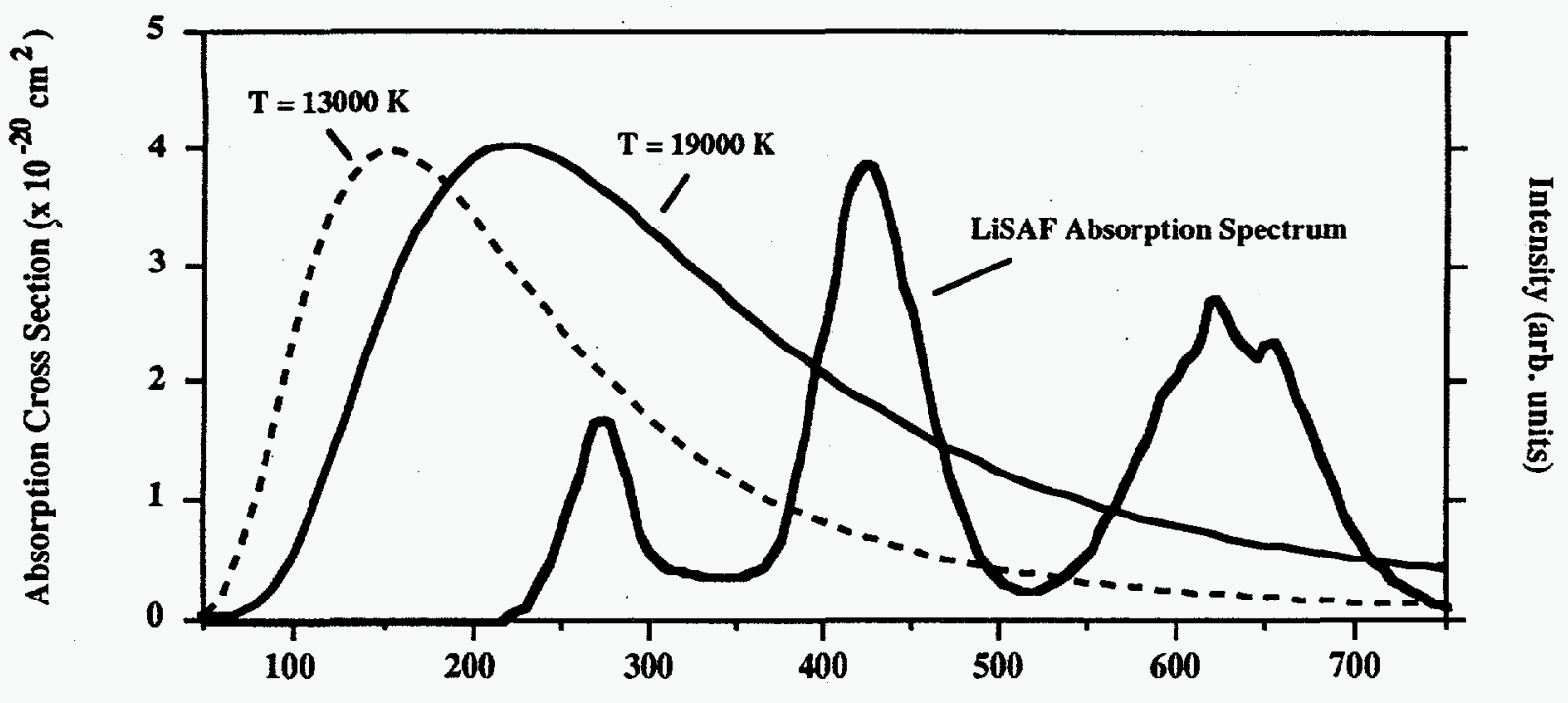

Wavelength (nm)

Figure 3: The dark solid line is the absorption spectrum of LiSAF (left axis). The calculated emission spectra of Xe flashlamps at $13000 \mathrm{~K}$ and $19000 \mathrm{~K}$ temperatures are compared to show the relative overlap with the LiSAF absorption.

The time dependence of the current density and the plasma temperature give rise to a changing emission spectrum. This results in a time-dependent pumping efficiency, $\gamma(r, t)$. The radial and temperature dependence of $\gamma(r, t)$ is found by calculating the pump light flux at a radius $r$ in the rod by assuming the surface of the rod is uniformly illuminated by the flashlamp light and that the light undergoes exponential absorption. The uniform illumination is integrated over the surface of the rod and this is then integrated over the normalized spectral distribution of the pump light, given by equation (2), $I_{n}(\lambda)=I(\lambda) / \int I(\lambda) d \lambda$, weighted by the absorption cross section. Since the rod length, $(l=100 \mathrm{~mm})$, is much greater than the rod radius, $(R=9.5 \mathrm{~mm})$, it is a good approximation to extend the limits of the integration over the length to $\pm \infty$ which yields for the pump efficiency:

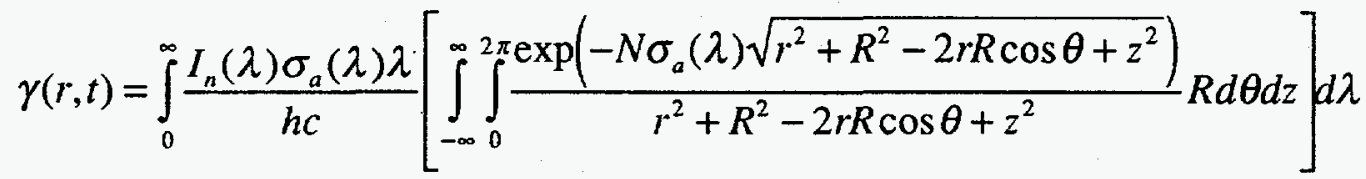

$\sigma_{a}(\lambda)$ is the absorption cross section of the LiSAF at a wavelength $\lambda$, and $\theta$ and $z$ are the coordinates of the rod surface. The calculated value of $\gamma(r, t)$ at the center of the rod $(r=0)$ and the peak of the flashlamp pulse as a function of discharged energy is shown in figure 4 . This pump efficiency drops by an appreciable amount as the pump energy is increased from 500 to $3000 \mathrm{~J}$.

The radial time dependent inversion density, $n(r, t)$, in the rod is then obtained by solving the rate equation:

$\frac{d n(r, t)}{d t}=-\frac{n(r, t)}{\tau}+\alpha_{c a v} \gamma(r, t) W(t) N$

Here $\tau$ is the upper state lifetime of the $\operatorname{LiSAF}(67 \mu \mathrm{s}), N$ is the $\mathrm{Cr}^{3+}$ ion density, and $W(t)$ is the normalized flashlamp light power per unit area incident on the rod surface. $\alpha_{c a v}$ is a fitting parameter that characterizes the over all efficiency of the pump cavity. The small signal gain experienced by a flat-top beam, calculated at the time when the inversion density is maximum, is then given by: 
$G=\frac{1}{\pi a^{2}} \int_{0}^{a} \exp \left(n(r, t) \sigma_{e} l\right) 2 \pi r d r$

with a beam radius of $a=7 \mathrm{~mm}$.

The calculated small signal gain as a function of pump energy is shown as a solid line in figure 2. For comparison, the gain calculated assuming a constant pump efficiency of $1.8 \%$ for all pump energies is shown as a dashed curve in figure 2 . Inclusion of the UV shifting in our model accurately predicts the observed small signal gain in this head for pump energies

Figure 4: Calculated pump efficiency using equation (3) as a function of discharged energy into eight flashlamps. The efficiency is evaluated at the peak of the flashlamp pulse for the rod center $(r=0)$.

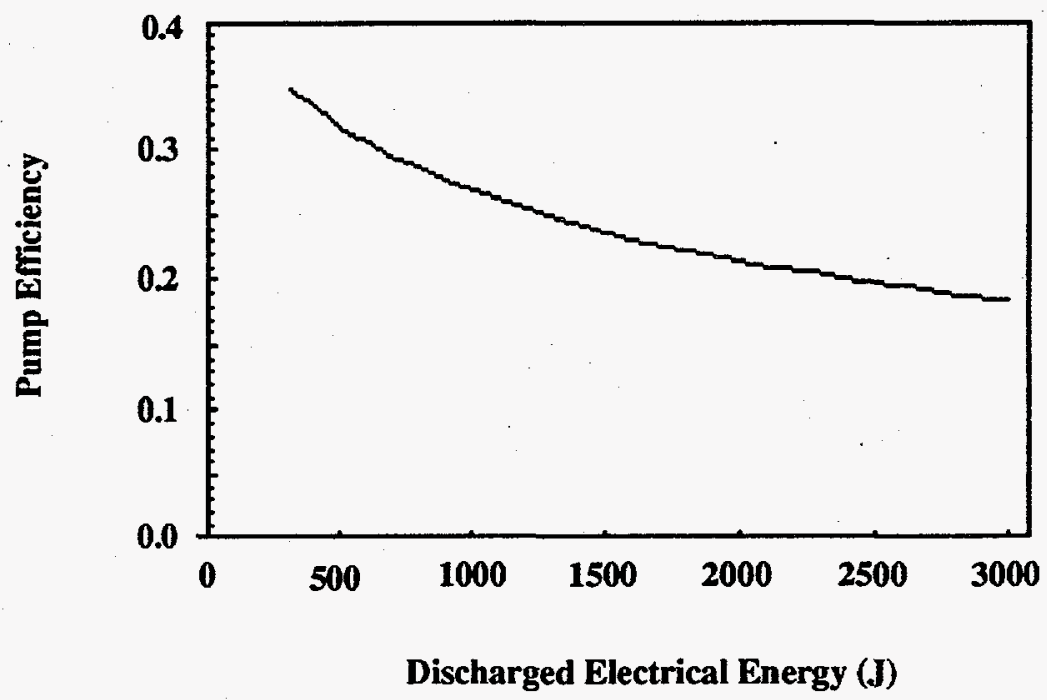

between $1 \mathrm{~kJ}$ and $3 \mathrm{~kJ}$ as well as the linear increase of the gain with increasing electrical pump energy. From these measurements we conclude that it is the UV shifting that sets the limit on flashlamp pump efficiency in large aperture LiSAF amplifiers. Furthermore, since the UV shift varies with the flashlamp current, further shortening of the flashlamp pulse width would increase the current density in the lamps and would not necessarily significantly improve the single pass gain performance. It is for this reason that we have chosen a flashlamp pulse that is $160 \mu \mathrm{s}$ instead of one that more closely matches the $67 \mu$ s lifetime of the LiSAF. The calculated gain in the $19 \mathrm{~mm}$ head as a function of flashlamp pulse width is shown in figure 5. This calculation suggests that a further decrease in the flashlamp pulse width results in very modest increases in gain. For example with $3 \mathrm{~kJ}$ of electrical energy into our eight flashlamps, shortening the pulse width to $70 \mu \mathrm{s}$ would result in only a

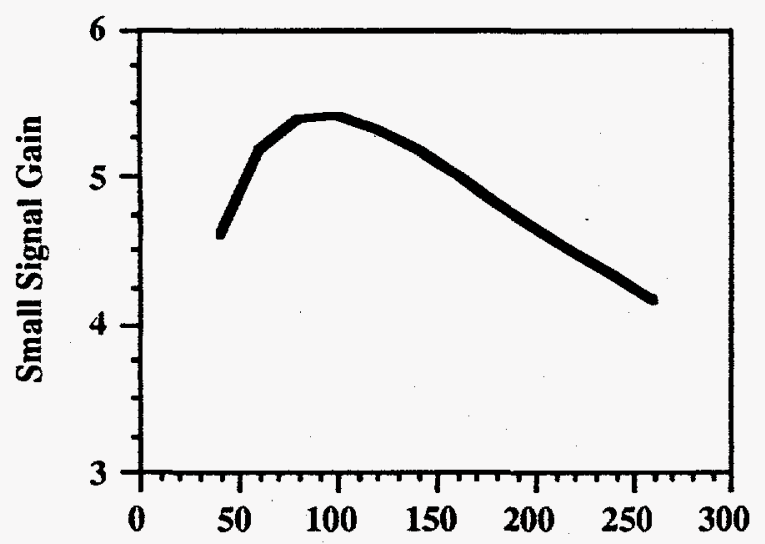

Figure 5: Calculated gain in the $19 \mathrm{~mm}$ head as a function of flashlamp pulse width for a fixed pump energy of $2800 \mathrm{~kJ}$.

Flashlamp pulse width ( $\mu s)$ 
slight increase in gain from 5 to 5.6. Below this value the calculated gain begins to drop. Concems about the lifetime of the flashlamps would also become important with shorter pulse widths. We operate our lamps at an explosion fraction of .25 above which Xe flashlamp lifetime begins to decrease rapidly. 16

Our $19 \mathrm{~mm}$ rod has a $\mathrm{Cr}^{3+}$ ion doping of $0.8 \%$ corresponding to an ion density of $1.0 \times 10^{20}$ ions $/ \mathrm{cm}^{3}$. This doping provides a good compromise between absorption efficiency and gain uniformity. The radially dependent small signal gain in the rod at an electrical pump energy of $2.7 \mathrm{~kJ}$ is shown in figure 6 . The gain varies from 3.5 at the center of the rod to 6.5 at the outer edge of the beam $(r=7 \mathrm{~mm})$ This gain variation, can be compensated by generating a signal pulse which exhibits an intensity profile opposite to the gain profile. We accomplish this by passing a truncated Gaussian beam through the preamplifiers resulting in beam exiting the final $19 \mathrm{~mm}$ amplifier which closely approximates a flat top profile. The resulting near field profile of the amplified beam after compression is shown in figure 7 .

\section{Pulse Recompression and Intensity Dependent Effects}

We have amplified the stretched pulse to over $1.7 \mathrm{~J}$ in the final amplifier. A vacuum spatial filter further expands the amplified beam to $5 \mathrm{~cm}$ for injection into a double-pass grating pair pulse compressor. The $1800 \mathrm{l} / \mathrm{mm}$ gratings were designed for high diffraction efficiency in the -1 order for $825 \mathrm{~nm}$ light. 19 The gratings each exhibit a diffraction efficiency of $~ 90 \%$ resulting is a compressor throughput of $63 \%$ and a recompressed pulse with an energy of up to $1.05 \mathrm{~J}$. The autocorrelation of the recompressed puise is shown in figure 8. The spectrum of the amplified pulse is shown in figure 9 . A Fourier transform of the amplified pulse spectrum indicates a transform limited pulse width of $125 \mathrm{fs}$ and an autocorrelation width of $190 \mathrm{fs}$ (shown in figure 8). This is in good agreement with the measured second-order autocorrelation width of 195 fs. This implies that the observed pulse is very nearly transform limited $(\Delta v \Delta \tau=.41)$ with a width of $125 \mathrm{fs}$. The intensity of the recompressed pulse at this point is in excess of $400 \mathrm{GW} / \mathrm{cm}^{2}$ and any propagation in air would result in significant self phase-modulation and spatial beam break-up. To circumvent this problem the compressor is enclosed in a chamber filled with argon which has a substantially lower nonlinear refractive index than air. Subsequent propagation of the recompressed pulse to the experiment is in argon.

With amplification of chirped pulses to energies of above $1 \mathrm{~J}$, the nonlinear phase accumulated before compression in the amplifier chain can severely distort the recompressed pulse shape. 11,20 This effect is noticeable at the highest extracted energies from the LiSAF system. Figure 10 shows the measured autocorrelation functions of the compressed pulse for two

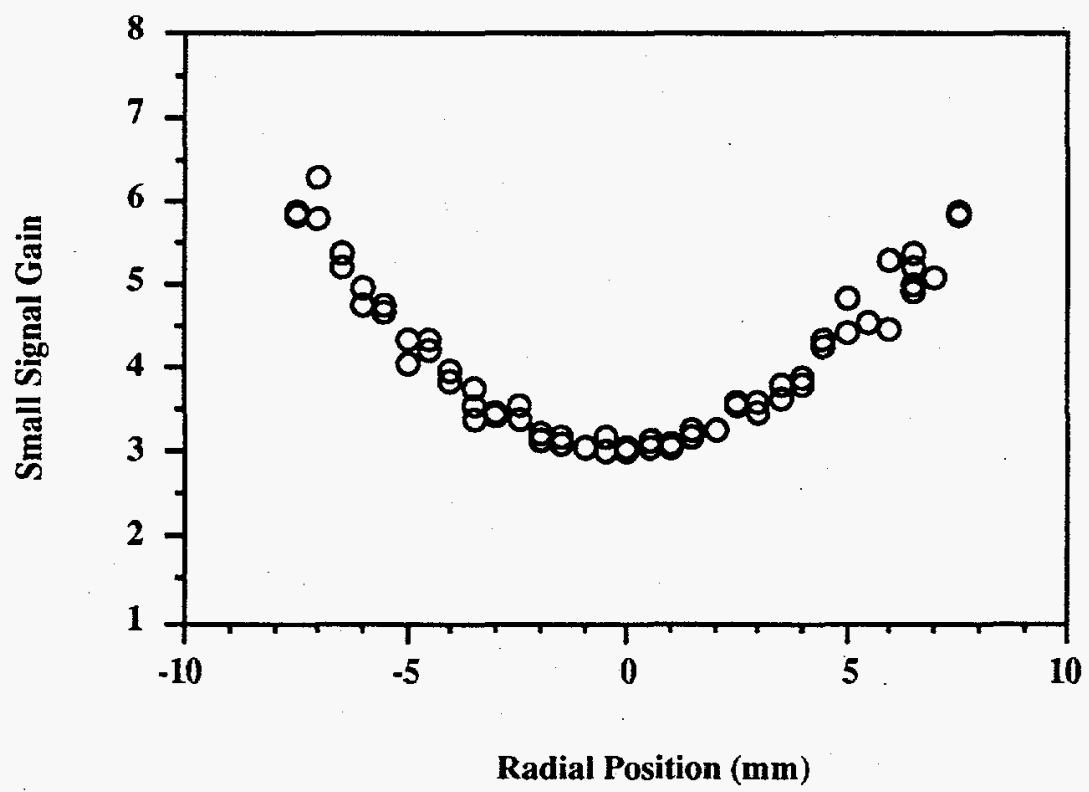

Figure 6: Measured small signal gain as a function of radial position in the $19 \mathrm{~mm}$ amplifier. 

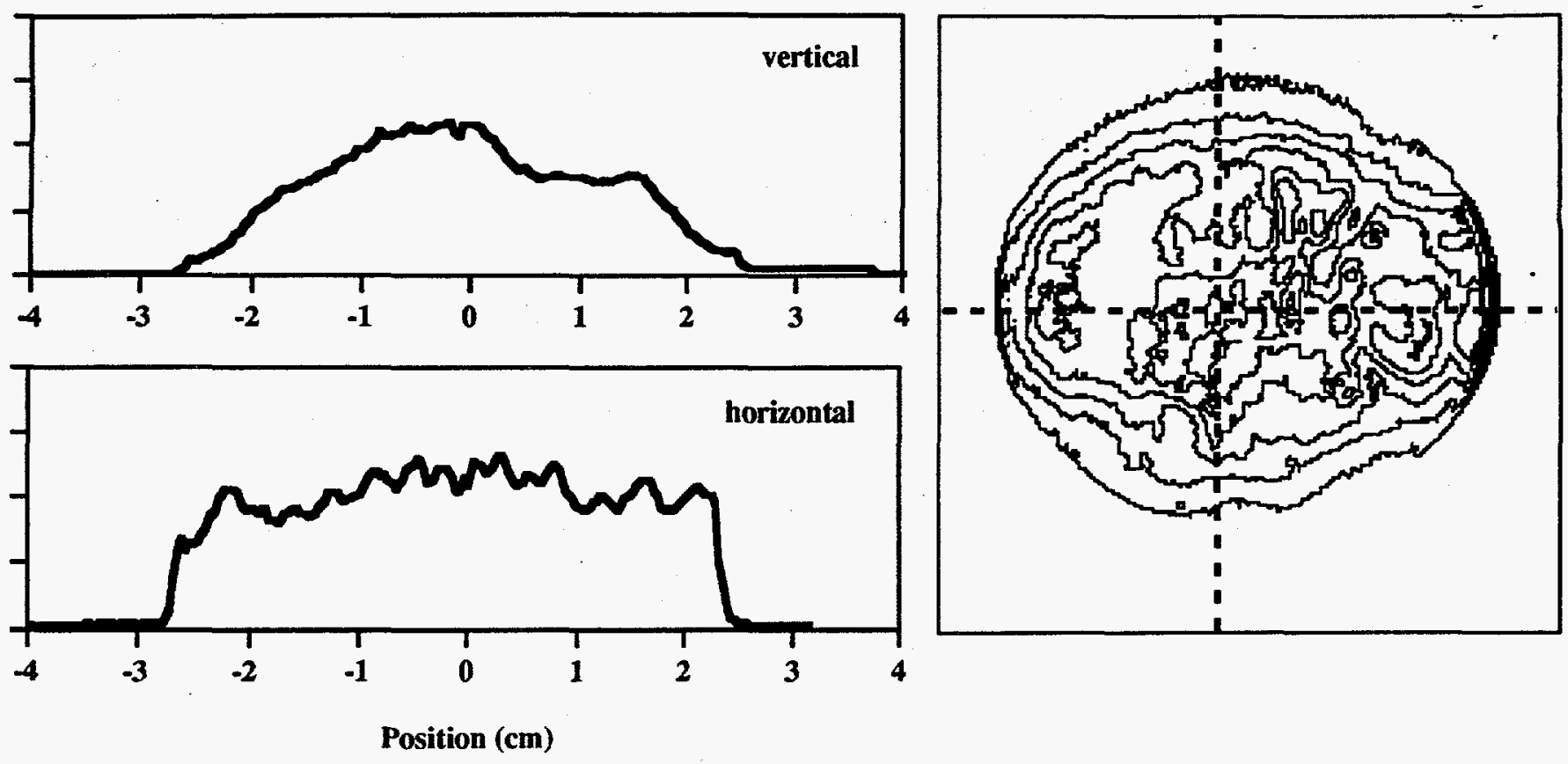

Figure 7: Near field beam profile after amplification to $1 \mathrm{~J}$.

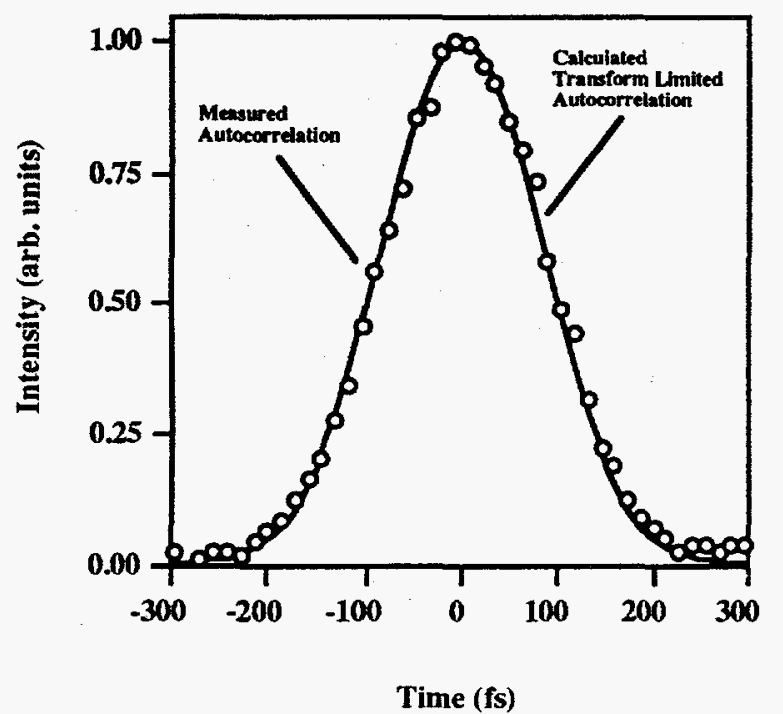

Figure 8: Measured autocorrelation trace after compression (circles). The FWHM of is 195 fs. The solid line is the calculated transform limited autocorrelation based on the measured spectrum.

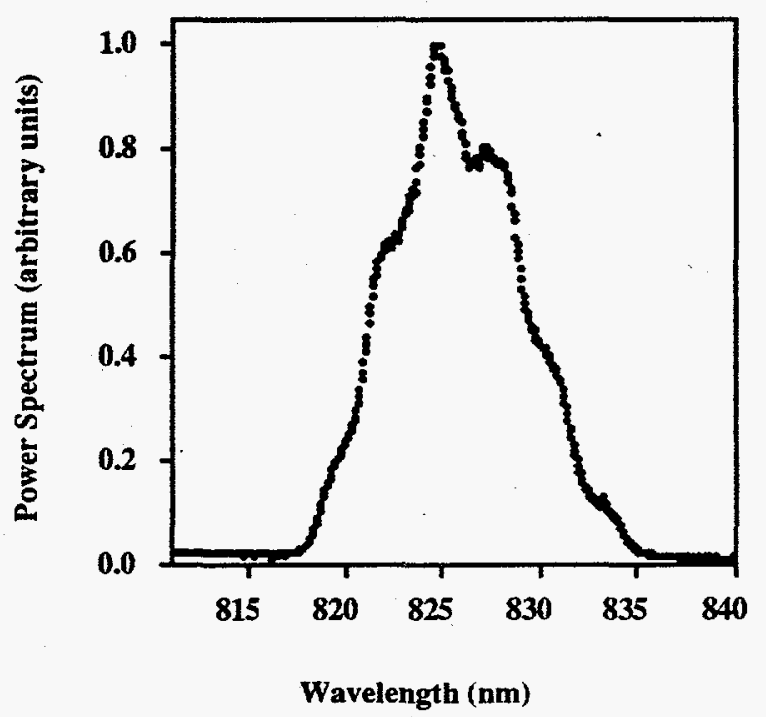

Figure 9: Measured spectrum of the amplified pulse.

values of the B-integral (total accumulated nonlinear phase). With a B-integral of $>1$ the recompressed pulse begins to broaden and develop wings. For this reason we operate with a B-integral of $\leq 1$. This effect can become a severe problem with chirped pulse systems operating with output energies of many joules. 20 


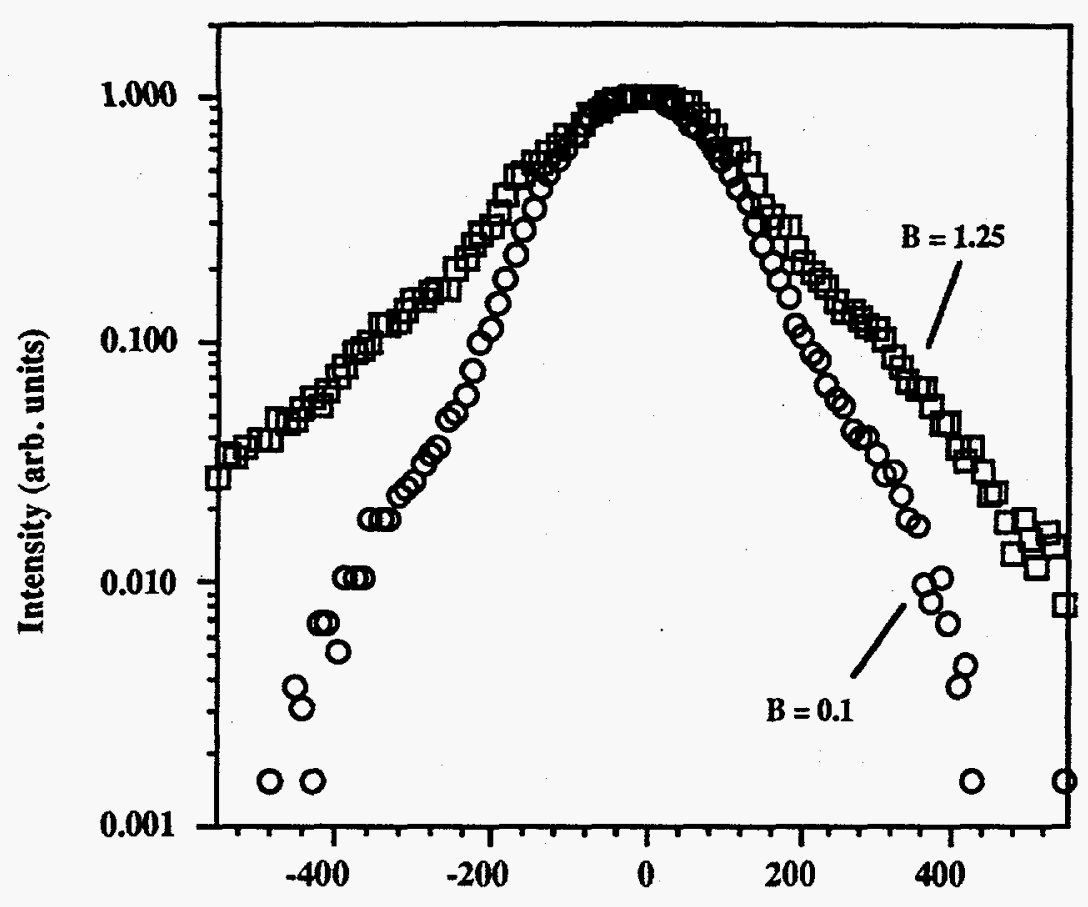

Figure 10: Measured autocorrelation after recompression with two values of accumulated nonlinear phase, $B$, in the amplifier chain; $\mathrm{B}=0.1$ (circles) and $\mathrm{B}=1.25$ (squares).

Time (fs)

Of equal importance to the peak power in high irradiance systems is the focusability of the beam. The $19 \times 110 \mathrm{~mm}$ Cr:LiSAF rod results in an overall peak to valley phase aberration of approximately $1 / 4$. This is not the limiting distortion in our system. Instead, it is the double pass in the first $9.5 \mathrm{~mm}$ amplifier resulting in a phase front distortion of $\approx 1 / 2$ that is responsible for the primary limit on focusibility. Far field and pinhole measurements of the focal spot produced with an $\mathrm{f} / 25$ lens indicate that roughly $55 \%$ of the focused pulse energy is contained in a near diffraction limited spot. These measurements were independent of pulse energy consistent with our estimate of a system nonlinear phase less than 1 radian at $1 \mathrm{~J}$ output from the compressor. The measured far field profile of the focal spot is shown in figure 11.

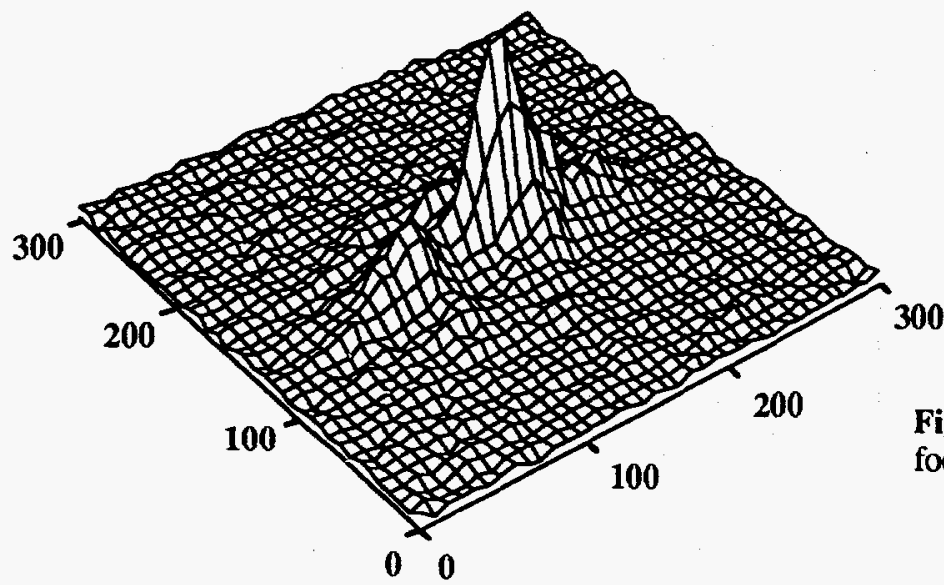

Position $(\mu \mathrm{m})$

Figure 11: Measured far field profile of the LiSAF laser focused with an $f / 25$ lens.

Ditmire et. al. 


\section{Conclusion}

In summary, we have developed a compact Cr.LiSAF laser system capable of producing femtosecond pulses with an energy of one joule. The system employs a flashlamp pumped, $19 \times 110 \mathrm{~mm}$ amplifier producing a radially averaged single pass gain of 4.8 for a pump energy of $3 \mathrm{~kJ}$. This permits amplification of stretched pulses to an energy of $1.7 \mathrm{~J}$ at a repetition rate of one shot every $20 \mathrm{sec}$. Recompression after amplification results in a transform-limited $125 \mathrm{fs}, 1.05 \mathrm{~J}$ pulse with a corresponding peak power of $8.5 \mathrm{TW}$. We have shown that flashlamp UV shifting sets an upper limit on the pump efficiencies that are possible with LiSAF. Though good pump efficiencies $(\sim 1.5 \%)$ are possible, further shortening of the flashlamp light or discharge of higher energies will not necessarily increase the gains possible in large aperture LiSAF amplifiers.

\section{Acknowledgements}

We would like to thank Russ Jones and Brad Golick for technical assistance, and we would like to acknowledge useful conversations with Howard Powell. This research was conducted for the U. S. Department of Energy by Lawrence Livermore National Laboratory under contract W-7405-Eng-48.

\section{References}

1 S. A. Payne, L.L. Chase, L. K. Smith, W.L. Kway, and H. W. Newkirk, "Laser Performance of $\mathrm{Cr}^{3+}$ :LiSrAIF6," J. Appl. Phys. 66, 1051 (1989).

2 A. Sullivan, H. Hamster, H. C. Kapteyn, S. Gordon, W. White, H. Nathel, R. J. Blair, and R. W. Falcone, "Multiterawatt, 100-fs Laser," Opt. Lett. 16, 1406 (1991).

3 J. D. Kmetec, J. J. Macklin, and J. F. Young, "0.5 TW, 125-fs Ti:sapphire Laser," Opt. Lett. 16, 1001 (1991).

4 C. P. J. Barty, C. L. Gordon III, and B. E. Lemoff, "Multiterawatt 30-fs Ti:sapphire Laser System," Opt. Lett. 19, 1442 (1994).

5 M. Stalder, B. H. T. Chai, and M. Bass, "Flashlamp Pumped Cr.LiSrAlF6 Laser," Appl. Phys. Lett., 58, 216 (1991)

6 T. Ditmire, and M. D. Perry, "Terawatt Cr:LiSrAIF6 Laser System," Opt. Lett. 18, 426 (1993).

7 P. Beaud, E. Miesak, Y-F. Chen, B. H. T. Chai, and M. C. Richardson, "110 fsec Fourier-Transform Limited Gaussian Pulses from a Cr:LiSAF Regenerative Amplifier," Opt. Comm. 95. 46 (1993).

8 W. E. White, L. van Woerkom, T. Ditmire, and M. D. Perry, "120-fs Terawatt Ti:Al2 $\mathrm{O}_{3} / \mathrm{Cr}$ :LiSrAlF6 Laser System, "Opt. Lett., 17 (1992).

9 P. Beaud, M. C. Richardson, E. J. Miesak, and B. H. T. Chai, "8-TW 90-fs Cr.LiSAF Laser," Opt. Lett. 18, 1550 (1993).

10 P. Beaud, M. C. Richardson, Y.-F. Chen, and B. H. T. Chai, "Optical Amplification Characteristics of Cr:LiSAF and Cr:LiCAF under Flashlamp-Pumping," IEEE J. Quant. Elec. QE-30, 1259 (1994).

11 M. D. Perry, T. Ditmire, and B. Stuart, "Self Phase-Modulation in Chirped Pulse Amplification," Opt. Lett., in press.

12 M. D. Perry, D. Strickland, T. Ditmire, and F.G. Patterson, "Cr:LiSrAlF, Regenerative Amplifier," Opt. Lett. 17, 604 (1992).

13 T. Ditmire, H. Nguyen, and M. D. Perry, "Design and Performance of a Multiterawatt Cr.LiSrAlF6 Laser System," J. Opt. Soc. Am. B, 11, 580 (1994).

14 J. B. Trenholme, and J. L. Emmett, "Xenon Flashlamp Model for Performance Prediction," Proceedings, Ninth International Conference on High Speed Photography, (SMPTE,1970), p. 299; G. J. Linford, R. A. Saroyan, J. B. Trenholme, and M. J. Weber, "Measurements and Modeling of Gain Coefficients for Neodymium Laser Glasses," IEEE J. Quant. Elec. QE-15, 510 (1979).

15 J. H. Kelly, D. C. Brown, and K. Teegarden, "Time Resolved Spectroscopy of Large Bore Xe Flashlamps for Use in Large Aperture Amplifiers," App. Opt. 19, 3817 (1980).

16 H. T. Powell, A. C. Erlandson, and K. S. Jancaitis, "Characterization of High Power Flashlamps and Application to Nd:Glass Laser Pumping," SPIE Proceedings Vol. 609, Conference on Flashlamp Pumped Laser Technology, pp. 78-94 (1986).

17 H. T. Powell, A. C. Erlandson, K. S. Jancaitis, J. E. Murray, "Flashlamp Pumping of Nd:Glass Disk Amplifiers," SPIE Proceedings Vol. 1277, Conference on High-Power Solid State Lasers and Applications, pp. 103-120 (1990).

18 J. P. Markiewicz, and J. L. Emmett, "Design of Flashlamp Driving Circuits," IEEE J. Quant. Elec. QE-2, 707 (1966). 
19 R. D. Boyd, J. Britten, D. Decker,"B. Shore, M. D. Perry and L. Li, "High-Efficiency Metallic Diffraction Gratings for Laser Applications," App. Opt. in press.

20 I. P. Mercer, "Ultra Short Pulse Generation For High Intensity Laser Application," Ph.D. Thesis, Department of Physics, Imperial College of Science, Technology and Medicine, London, SW7 2BZ, UK. 